[3] L. Kurz, "Nonparametric detectors based on partition tests," in Nonparametric Methods in Communications. P. Papantoni-Kazakos and D. Kazakos, Eds. New York: Marcel Dekker: 1977, pp. $73-143$.

[4] S. A. Kassam, "Conditional tests in nonparametric detection, $\because$ in Nonparametric Methods in Communications, P. Papantoni-Kazakos and D. Każakos, Eds. New York: Marcel Dekker, 1977, pp. $145-201$.

[5] J. G. Shin and S. A. Kassam. "Multilével coincidence correlators for random 'signal detection." IEEE Trans. Inform. Theory, vol. IT-25., pp. 47-53; Jan. 1979

[6] P. Kersten and L. Kurz, "Bivariate $m$-interval classifiers with application to edge detection." Inform. Contr., vol. 34, pp. 152I68, Ju'ne 1977.

[7] S. A. Kassam. "Optimum quantization for signal detection." IEEE Trans: Commun.. vol. COM-25, pp. 479-484, May 1977.

[8] H. V. Poor and J. B. Thomas, "Application of Ali-Silvey distance measures in the design of generalized quantizers for binary decision systems," 'IEEE Trans.' Commun:, vol. COM-25, pp. '893-900, Sept. 1977.

[9] H. V. Poor and Y. Rivani, "Input amplitude compression in digital signal-detection systems," IEEE Trans. Commun., vol. COM-29, pp. 707-710, May 1981 .

[10] T. L. Lim, "Noncoherent digital matched filters: Multibit quantization." IEEE Trans. Commun., vol. COM-26, pp. 409419, Apr. 1978.

[11] J. Capon, "On the asymptotic efficiency of locally optimum detectors," IRE Trans. Inform. Theory, vol. IT-7, pp. 67-71, Apr. 1961 .

\section{GMSK Modulation for Digital Mobile Radio Telephony}

\author{
KẠZUAKI MUROTA, MEMBER, IEEE, AND KENKICHI HIRADE, \\ MEMBER, IEEE
}

\begin{abstract}
This paper is concerned with digital modulation for future móbile radio telephone services. First, the specific requirements on the digital modulation for mobile radio use are described. Then, premodulation Gaussian filtered minimum shift keying (GMSK) with coherent detection is proposed as an effective digital modulation for the present purpose, and its fundamental properties are clarified with the aid of machine computation. The constitution of modulator and demodulator is then discussed from the viewpoints of mobile radio applications. The superiority of this modulation is supported by some experimental test results.
\end{abstract}

\section{INTRODUCTION}

It is well known that voice transmission in many VHF and UHF mobile radio telephone systems has usually been made by using a single-channel-per-carrier (SCPC) analog FM transmission technique. However, in order to provide highly secure voice and/or high-speed data transmission by the use of largescale integrated (LSI) transceivers, digital mobile radio trans-

Paper approved by the Editor for Communication Theory of the IEEE Communications Society for publication after presentation at 29th IEEE Vehicular Technology Conference, Chicago, IL; March 1979. Manuscript received May 28,1980; revised January 5, 1981.

The authors are with the Yokosuka Electrical Communication Laboratory, Nippon Telegraph and" Telephone Public Corporation, Kanagawa-Ken, Japan. mission is currently being studied [1]-[7]. While digital transmission can surely bring many advantages, some technical problems must be solved. This paper is concerned with a digital modulation for future mobile radio communications.

From the viewpoint of mobile radio use, the out-of-band radiation power in the adjacent channel should be generally suppressed $60-80 \mathrm{~dB}$ below that in the desired channel. So as to satisfy this severe requirement, it is necessary to manipulate the RF output signal spectrum. Such a spectrum manipulation cannot usually be performed at the final RF stage in the multichannel SCPC transceivers because the transmitted $R F$ frequency is variable. The'refore, intermediate-frequency (IF) or baseband filtering with frequency up conversion is mostly used. However, when such a spectrum-manipulated signal is translated up and passed through a nonlinear class- $C$ power amplifier, the required spectrum manipulation should not be violated by the nonlinearities. In order to mitigate the impairments, some narrow-band digital modulation schemes with constant or less fluctuated envelope property have been researched $[8]-[10]$.

In this paper, premodulation Gaussian filtered minimum shift keying (GMSK) with coherent detection is proposed as an effective digital modulation for the present purpose, and its fundamental properties are analyzed with the aid of machine computation. The relationship between out-of-band radiation suppression and bit-error-rate (BER) performance is made clear. Constitution of the modulator and demodulator is then discussed. The superiority of this modulation is supported by some experimental test results.

\section{GMSK MODULATION}

\section{A. Spectrum Manipulation of MSK}

Minimum shift keying (MSK), which is binary digital FM with a modulation index of 0.5 , has the following good properties: constant envelope, relatively narrow bandwidth, and coherent detection capability [11]-[13]. However, it does not satisfy the severe requirements with respect to outof-band radiation for SCPC mobile radio. MSK can be generated by direct FM modulation. As is easily found, the output power spectrum of MSK can be manipulated by using a premodulation low-pass filter (LPF), keeping the constant envelope property, as shown in Fig. 1. To make the output power spectrum compact, the premodulation LPF should have the following properties:

1) narrow band width and sharp cutoff

2) lower overshoot impulse response

3) preservation of the filter output pulse area which corresponds to a phase shift $\pi / 2$.

Condition 1) is needed to suppress the high-frequency components, 2) is to protect against excessive instantaneous frequency deviation, and 3 ) is for coherent detection to be applicable as simple MSK.

Generally, the introduction of the premodulation LPF violates the minimum frequency spacing constraint and the fixed-phase constraint of MSK. However, the above two constraints are not intrinsic requirements for effective coherent binary FM with modulation index 0.5 . Such a premodulationfiltered MSK signal can be detected coherently because its 


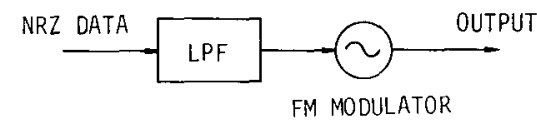

Fig. 1. Premodulation baseband-filtered MSK. and

pattern-averaged phase-transition trajectory does not deviate from that of simple MSK.

\section{B. Fundamental Properties of GMSK}

A Gaussian LPF satisfies all the above-described characteristics. Consequently, the modified MSK modulation using a premodulation Gaussian LPF can be expected to be an excellent digital modulation technique for the present purpose. Such a modified MSK is named Gaussian MSK or GMSK in connection with Gaussian low-pass filtering. Let us now investigate the GMSK modulation from various aspects.

Output Power Spectrum: Fig. 2 shows the machine-computed results of the output power spectrum of the GMSK signal versus the normalized frequency difference from the carrier center frequency $\left(f-f_{c}\right) T$ where the normalized $3 \mathrm{~dB}$ down bandwidth of the premodulation Gaussian LPF $B_{b} T$ is a parameter. The spectrum for GMSK with $B_{b} T=0.2$ is nearly equal to that of TFM.

The effective variable parameter $B_{b} T$ can be selected by the system designer considering overall spectrum efficiency of the cellular zone structure.

Fig. 3 shows the machine-computed results of the fractional power in the desired channel versus the normalized bandwidth of the predetection rectangular bandpass filter (BPF) $B_{i} T$. Table $I$ shows the occupied bandwidth for the prescribed percentage of power where $B_{b} T$ is also a variable parameter. For comparison, the occupied bandwidth of TFM is also shown in Table I.

Fig. 4 shows the machine-computed results of the ratio of the out-of-band radiation power in the adjacent channel to the total power in the desired channel where the normalized channel spacing $f_{S} T$ is taken as the abscissa and both channels are assumed to have the ideal rectangular bandpass charácteristics with $B_{i} T=1$. The situation of $f_{s} T=1.5$ and $B_{i} T=1$ corresponds to the case of $f_{s} \cong 25 \mathrm{kHz}$ and $B_{i}=16 \mathrm{kHz}$ when $f_{b}=1 / T=16 \mathrm{kbits} / \mathrm{s}$. From Fig. 4 , it is found that the GMSK with $B_{b} T=0.28$ can be adopted as the digital modulation for conventional VHF and UHF SCPC mobile radio communications without carrier frequency drift where the ratio of out-ofband radiation power in the adjacent channel to the total power in the desired channel must be lower than $-60 \mathrm{~dB}$. When a certain amount of carrier frequency drift (for example $\Delta f= \pm 1.5 \mathrm{kHz}$ ) exists, $B_{b} T=0.2$ is needed.

$B E R$ Performance: Let us now consider the theoretical BER performance of GMSK modulation using coherent detection in the presence of additive white Gaussian noise.

Since the GMSK modulation of interest is a certain kind of binary digital modulation, its BER performance bound in the high SNR condition is approximately represented as

$$
P_{e}=\frac{1}{2} \operatorname{erfc}\left(\frac{d_{\min }}{2 \sqrt{N_{0}}}\right)
$$

where $N_{0}$ is the power spectrum density of the additive white Gaussian noise and erfc( ) is the complementary error func-

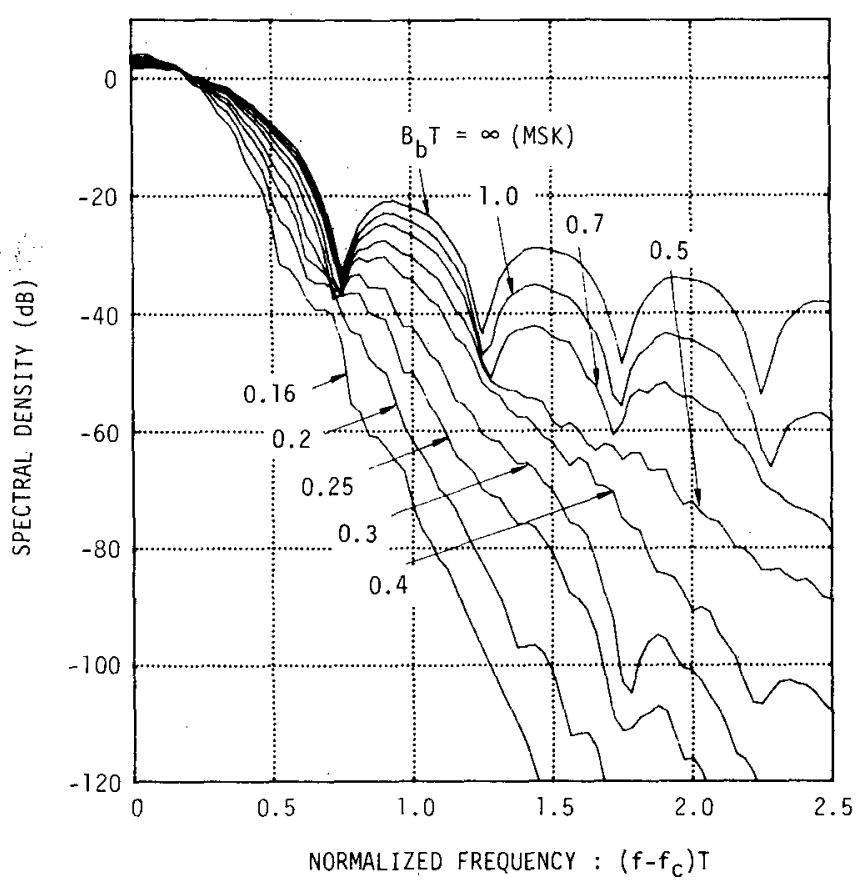

Fig. 2. Power spectra of GMSK.

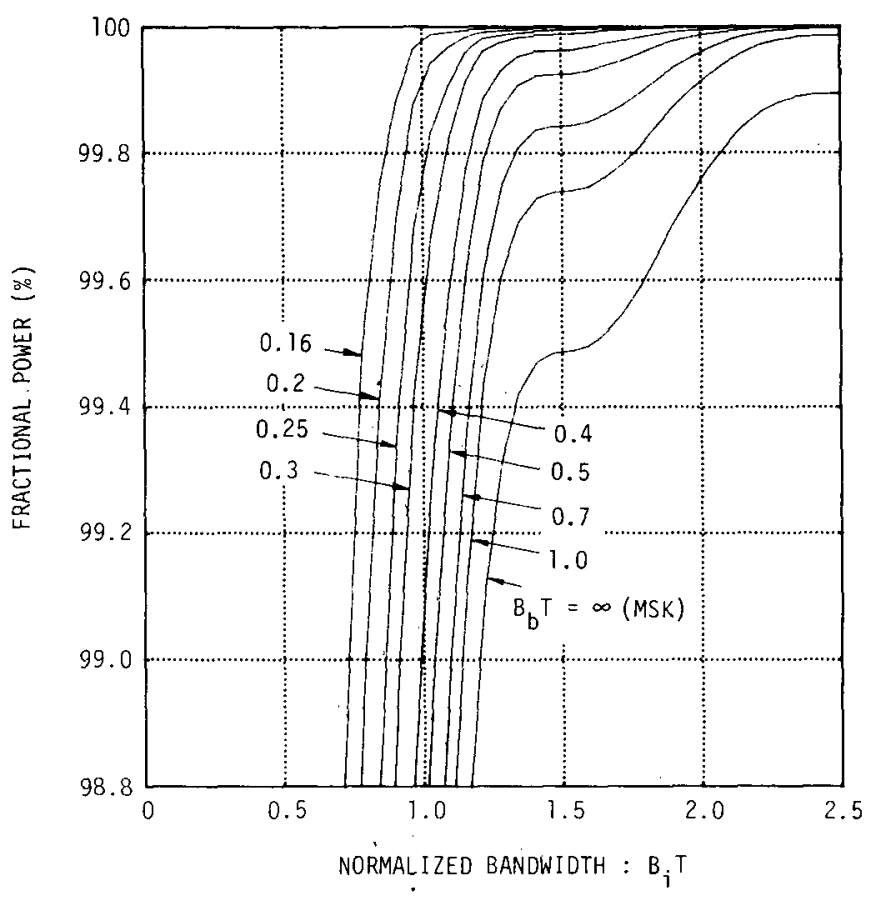

Fig. 3. Fractional power ratio of GMSK. 
TABLE I

OCCUPIED BANDWIDTH CONTAINING A GIVEN PERCENTAGE POWER

\begin{tabular}{|c|c|c|c|c|}
\hline$B_{b}^{\top} \%$ & 90 & 99 & $99: 9$ & 99.99 \\
\hline 0.2 & 0.52 & 0.79 & 0.99 & 1.22 \\
\hline 0.25 & 0.57 & 0.86 & 1.09 & 1.37 \\
\hline 0.5 & 0.69 & 1.04 & 1.33 & 2.08 \\
\hline MSK & 0.78 & 1.20 & 2.76 & 6.00 \\
\hline TFM & 0.52 & 0.79 & 1.02 & 1.37 \\
\hline
\end{tabular}

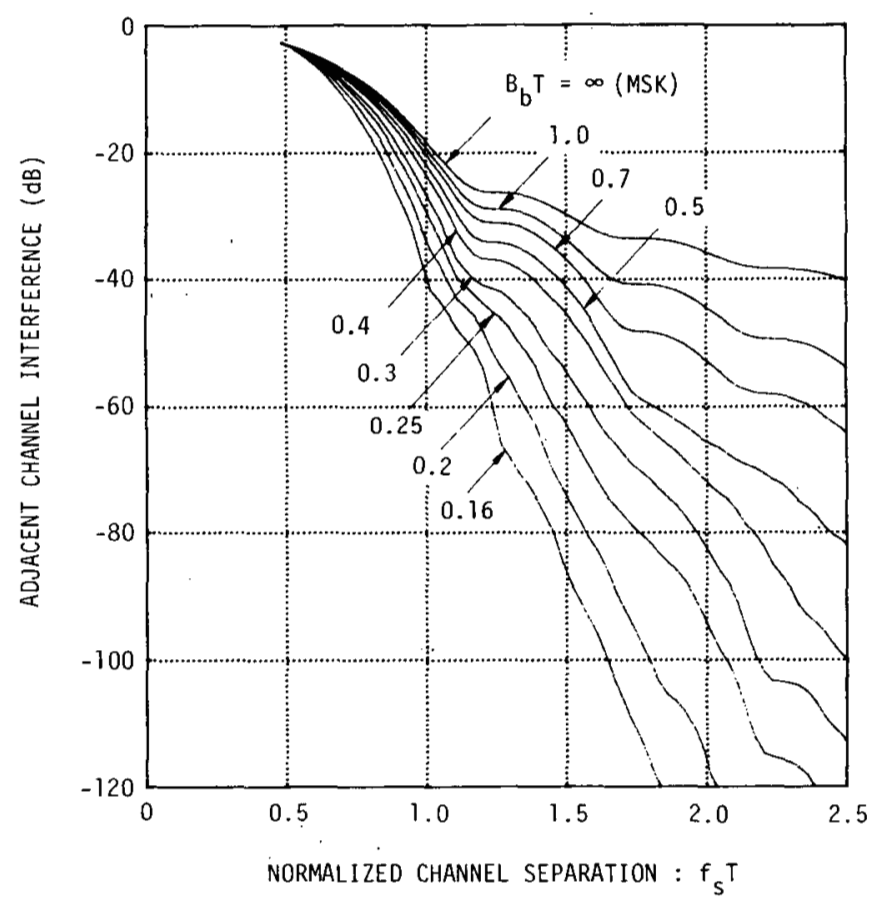

Fig. 4. Adjacent channel interference of GMSK.

tion given by

$$
\operatorname{erfc}(x)=\frac{2}{\sqrt{\pi}} \int_{x}^{\infty} \exp \left(-u^{2}\right) d u
$$

Furthermore, $d_{\mathrm{m} \text { in }}$ is the minimum value of the signal distance $d$ between mark and space in Hilbert space observed during the time interval from $t_{1}$ to $t_{2}$ and $d$ is defined by

$$
\dot{d}^{2}=\frac{1}{2} \int_{t_{1}}^{t_{2}}\left|u_{m}(t)-u_{s}(t)\right|^{2} d t
$$

where $u_{m}(t)$ and $u_{s}(t)$ are the complex signal waveforms corresponding to the mark and the space transmissions, respectively.

While the BER performance bound given by (1) is attained only when the ideal maximum likelihood detection is adopted, it gives an approximate solution for the ideal BER performance of GMSK modulation with coherent detection.

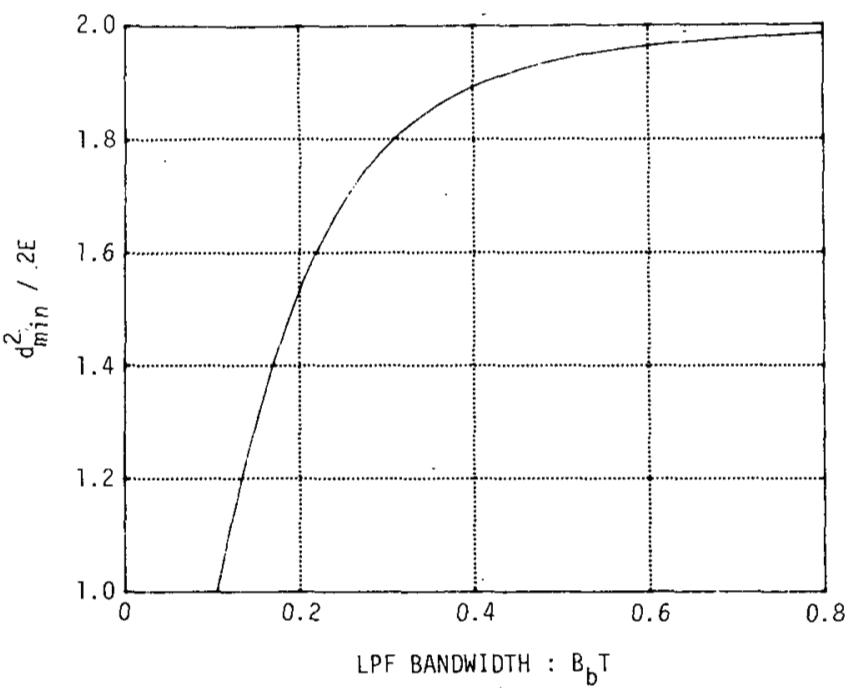

Fig. 5. Normalized minimum signal distance of GMSK.

Fig. 5 shows the machine-computed results for $d_{\mathrm{m} \text { in }}$ of the GMSK signal versus $B_{b} T$ where $E_{b}$ denotes the signal energy per bit defined by

$$
E_{b}=\frac{1}{2} \int_{0}^{T}\left|u_{m}(t)\right|^{2} d t=\frac{1}{2} \int_{0}^{T}\left|u_{s}(t)\right|^{2} d t .
$$

In the case $B_{b} T \rightarrow \infty$, which corresponds to the simple MSK signal, Fig. 5 yields $d_{\mathrm{min}}=2 \sqrt{E_{b}}$, which is that of antipodal transmission. It is noticed that the meaningful observation time interval for the GMSK signal $t_{2}-t_{1}$ may be made longer than $2 T$, which corresponds to that for the simple MSK signal, due to the intersymbol interference (ISI) effect on the phase transitions.

Substituting the machine-computed results of $d_{\mathrm{min}}$ into (1), the BER performance of the GMSK modulation with coherent detection is obtained. Fig. 6 shows the performance degradation of GMSK from antipodal transmission due to the ISI effect of the premodulation LPF. This figure shows that the performance degradation is small and that the required $E_{b} / N_{0}$ of GMSK with $B_{b} T=0.25$ does not exceed more than $0.7 \mathrm{~dB}$ compared to that of antipodal transmission.

\section{IMPLEMENTATION}

\section{A. Modulator}

The simple and easy method is to modulate the frequency of VCO directly by the use of baseband Gaussian pulse stream, as shown in Fig. 1. However, this modulator has the weak point that it is difficult to keep the center frequency within the allowable value under the restriction of maintaining the linearity and the sensitivity for the required FM modulation. Such a weak point can be removed by the use of an elaborate PLL modulator with a precisely designed transfer characteristics or an orthogonal modulator with digital waveform generators [14]. Instead of such a modulator, a $\pi / 2$-shift binary PSK (BPSK) modulator followed by a suitable PLL phase smoother, as shown in Fig. 7 , is considered to be a prominent alternative where the transfer characteristics of this PLL are also designed for the output power spectrum to satisfy the required condition. 


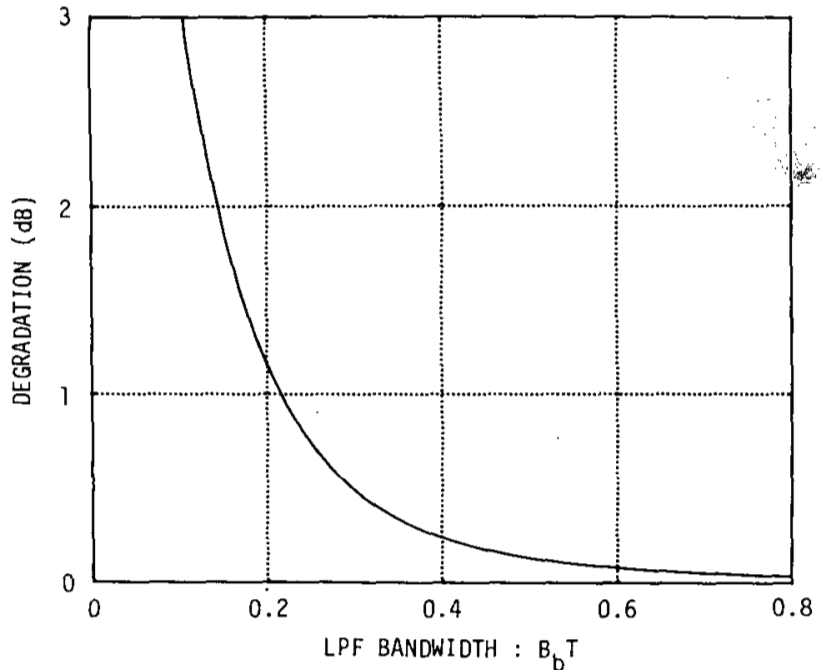

Fig. 6. Theoretical $E_{b} / N_{0}$ degradation of GMSK.

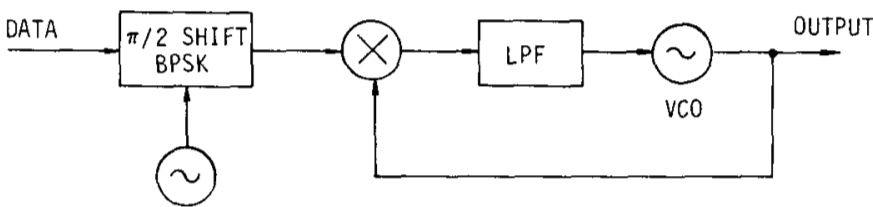

Fig. 7. PLL-type GMSK modulator.

\section{B. Demodulator}

Similar to the simple MSK or TFM system, the orthogonal coherent detector is also applicable for the GMSK system. When realizing such an orthogonal coherent detector, one of the most important and difficult problems is how to recover the reference carrier and the timing clock. The most typical method is de Buda's one [12]. In his method, the reference carrier is recovered by dividing by four the sum of the two discrete frequencies contained in the frequency doubler output and the timing clock is directly recovered by their difference. Remembering that the action of the well-known Costas loop as a carrier recovery circuit for BPSK systems is equivalent to that of a PLL with a frequency doubler [15], de Buda's method is realized by the equivalent one shown in Fig. 8(a). This modified method can easily be implemented by conventional digital logic circuits and its configuration is also shown in Fig. 8(b). In this configuration, two $D$ flip-flops act as the quadrature product demodulators and both of the ExclusiveOr logic circuits are used for the baseband multipliers. Furthermore, the mutually orthogonal reference carriers are generated by the use of two $D$ flip-flops, and the VCO center frequency is then set equal to the four times carrier center frequency. This configuration is considered to be especially suitable for the mobile radio unit which must be simplified, miniaturized, and economized.

\section{EXPERIMENTS}

\section{A. Test System}

Fig. 9 shows the block diagram of the experimental test system where the carrier frequency and the bit rate are $f_{c}=$ $70 \mathrm{MHz}$ and $f_{b}=16 \mathrm{kbits} / \mathrm{s}$, respectively. A pseudonoise (PN) pulse sequence with a repetition period of $N=\left(2^{15}-1\right)$ bits is generated by the 15-stage feedback shift register (FSR) and is used as a test pattern signal. After passing through a pre-

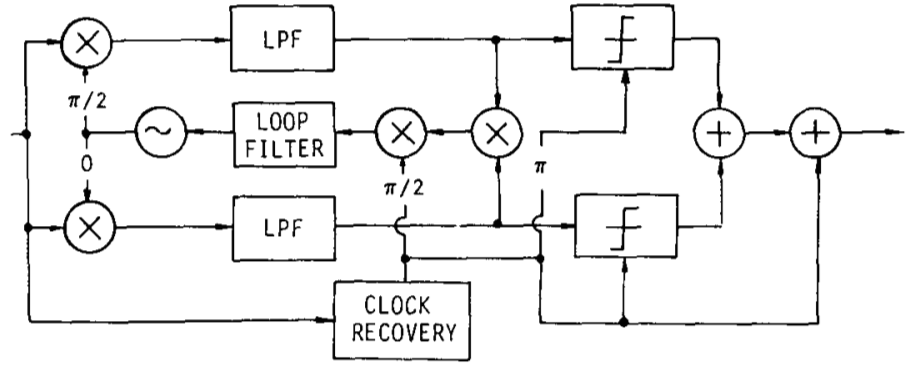

(a)

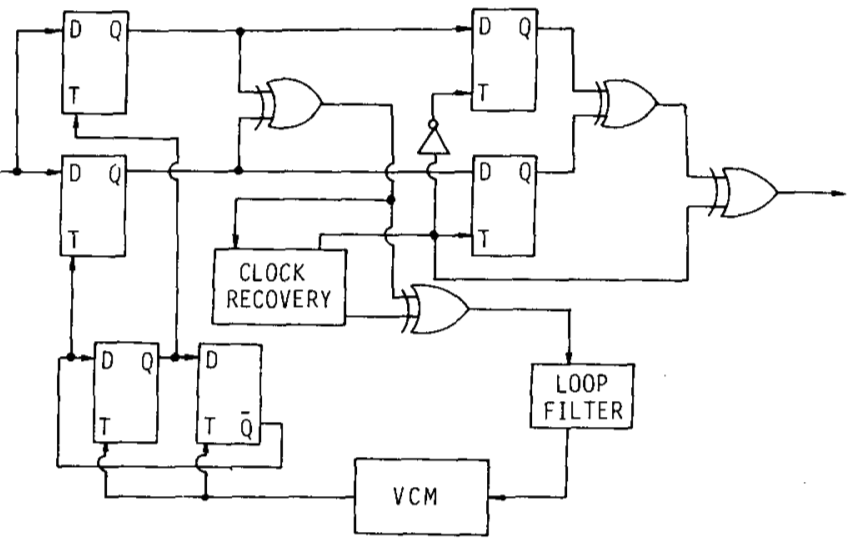

(b)

Fig. 8. Orthogonal coherent detector for MSK/GMSK. (a) Analog type. (b) Digital type.

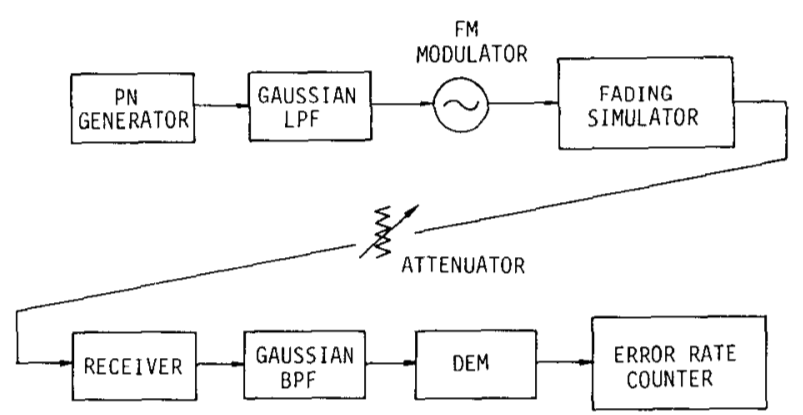

Fig. 9. Block diagram of experimental test system.

modulation Gaussian LPF having a variable bandwidth $B_{b}$, the PN sequence is put into the synthesized RF signal generator having an external FM modulation capability. The frequency deviation of the RF signal generator is set equal to $\Delta f_{d}=$ $\pm 4 \mathrm{kHz}$, which corresponds to the MSK condition for the $16 \mathrm{kbits} / \mathrm{s}$ transmission. Then the GMSK signal of our choice is obtained as the RF signal generator output, and is transmitted into the receiver via the Rayleigh fading simulator [16]. Predetection bandpass filtering in the receiver is performed by the precisely designed Gaussian bandpass crystal filter. The bandpass-filtered output is demodulated by the digital orthogonal coherent detector shown in Fig. 8. The regenerated output is fed into the error-rate counter for the BER measurement.

\section{B. Power Spectrum and Eye Pattern}

Fig. 10 shows the measured power spectra of the RF signal generator output when $B_{b} T$ is a variable parameter. It is clearly seen that the measured results agree well with the machine-computed ones shown in Fig. 2. Moreover, GMSK with $B_{b} T=0.25$ is shown to satisfy the severe requirements 


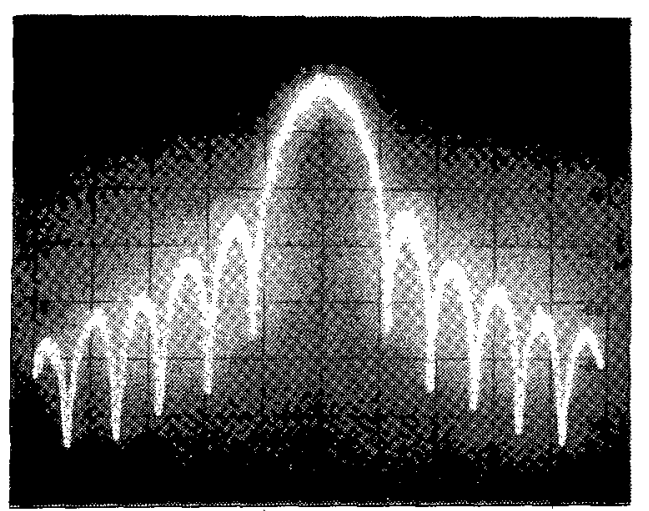

$$
\begin{gathered}
{ }_{b}{ }^{T}=\infty \\
(M S K)
\end{gathered}
$$

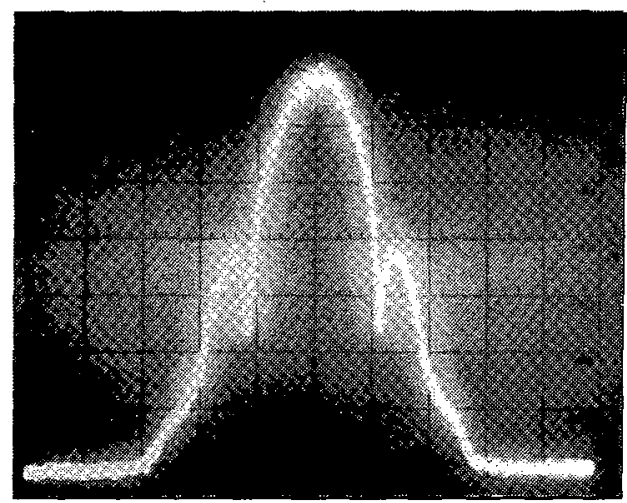

$$
B_{b} T=0.5
$$$$
B_{b} T=0.25
$$
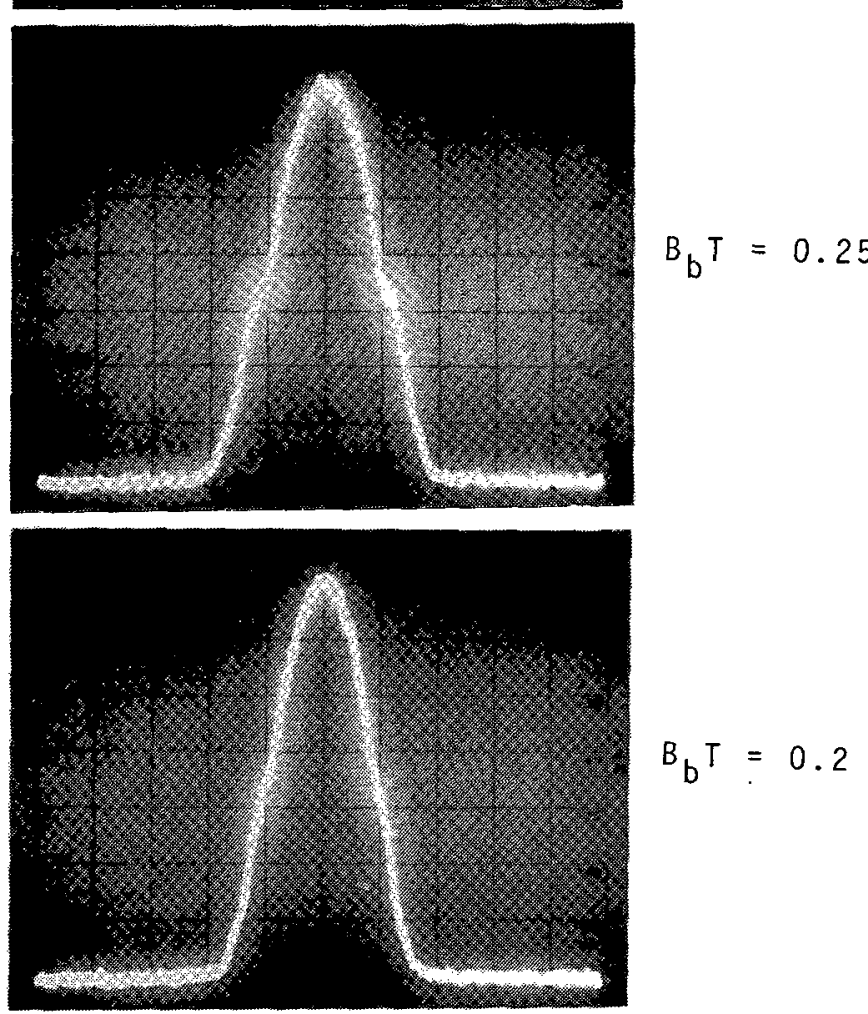

$$
B_{b} T=0.2
$$

Fig. 10. Measured power spectra of GMSK $(V: 10 \mathrm{~dB} /$ div., $H: 10$ $\mathrm{kHz} /$ div.).

of the out-of-band radiation of SCPC mobile radio communications. The corresponding eye pattern measured at the premodulation Gaussian LPF output is shown in Fig. 11. This figure shows that the above satisfactory performance of the out-of-band radiation can only be attained by the sacrifice of introducing severe ISI effects into the baseband waveform of the FM modulator input. It might be feared that such a severe

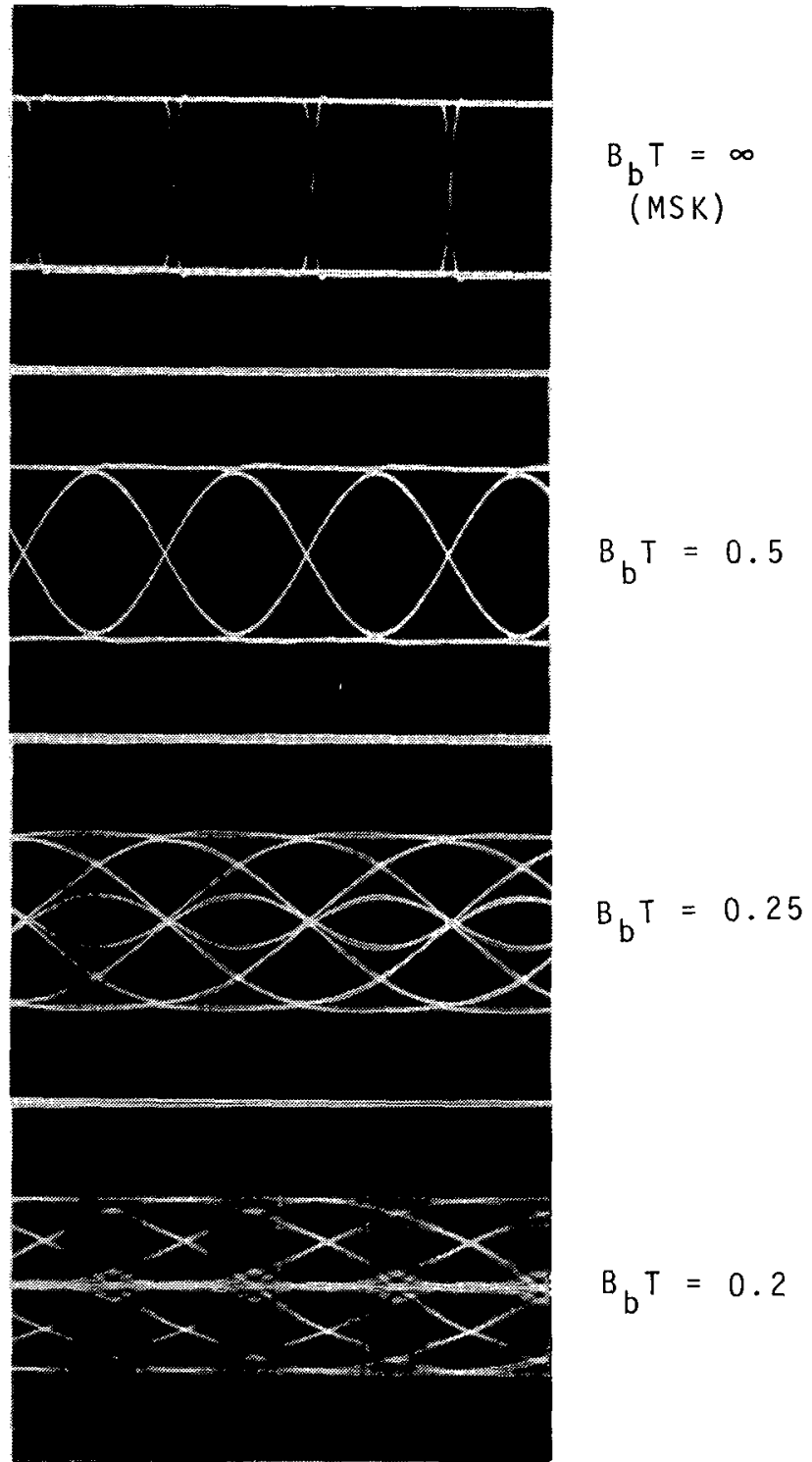

Fig. 11. Instantaneous frequency variation of GMSK.

ISI effect causes inferior transmission performance. However, this misgiving is happily unwarranted because the dernodulator output of GMSK with $B_{b} T=0.25$ degrades only slightly from that of simple MSK. It is easily found from Fig. 12 which shows the respective eye patterns measured by the analog-type orthogonal coherent detector shown in Fig. 8(a). It is also certified from the BER performance test results described later.

\section{Static BER Performance}

Fig. 13 shows experimental test results for static BER performance in the nonfading environment where the normalized 3 dB-down bandwidth of the premodulation Gaussian LPF, $B_{b} T$, is a variable parameter and the normalized $3 \mathrm{~dB}$-down bandwidth of the predetection Gaussian BPF is $B_{i} T \cong 0.63$, i.e., $B_{i}=10 \mathrm{kHz}$ for $f_{b}=1 / T=16 \mathrm{kbits} / \mathrm{s}$. The condition $B_{i} T \cong 0.63$ is nearly optimum, as shown in Fig. 14. From Fig. 13 , performance degradation of GMSK with $B_{b} T=0.25$ relative to simple MSK is found to be only $1.0 \mathrm{~dB}$. Moreover, the measured static BER performance of simple MSK degrades by $0.7 \mathrm{~dB}$ from the theoretical one of ideal antipodal binary 

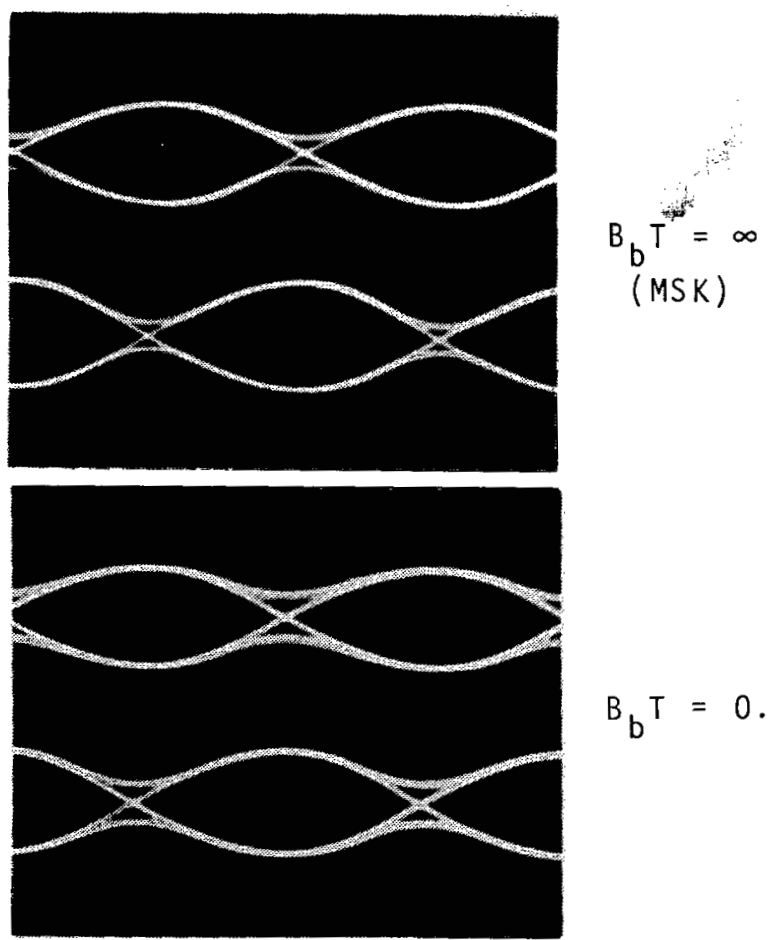

$B_{b} T=0.5$
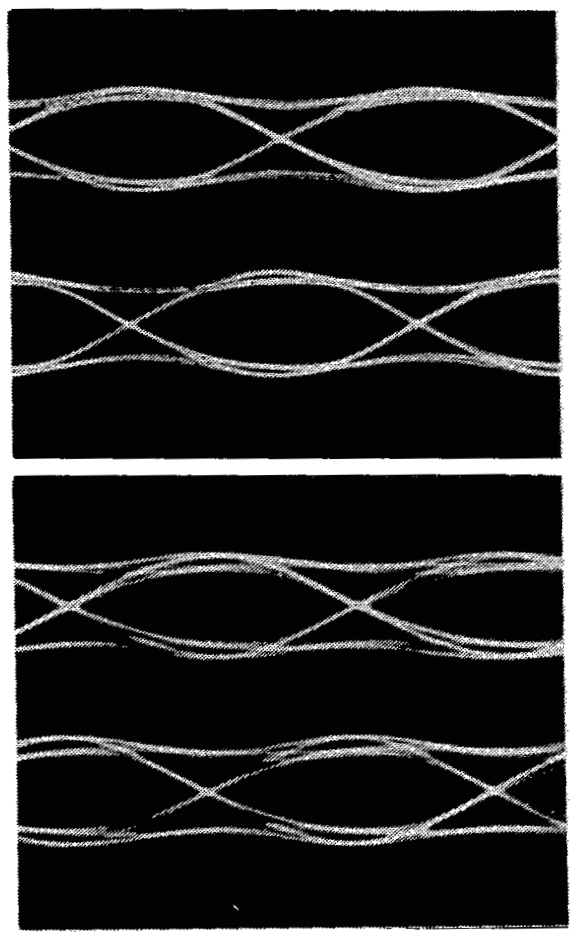

$B_{b}{ }^{\top}=0.2$

Fig. 12. GMSK eye patterns demodulated by orthogonal coherent detector.

transmission system. If $\gamma$ denotes the received signal energyto-noise density ratio, i.e., $E_{b} / N_{0}$, the measured static BER performance in the nonfading environment can be approximated as

$$
P_{e}(\gamma) \cong \frac{1}{2} \operatorname{erfc}(\sqrt{\alpha \gamma})
$$

where erfe( ) is the complementary error function given by (2) and $\alpha$ is a constant parameter determined as

$$
\alpha \cong \begin{cases}0.68 & \text { for GMSK with } B_{b} T=0.25 \\ 0.85 & \text { for simple MSK }\left(B_{b} T \rightarrow \infty\right) .\end{cases}
$$

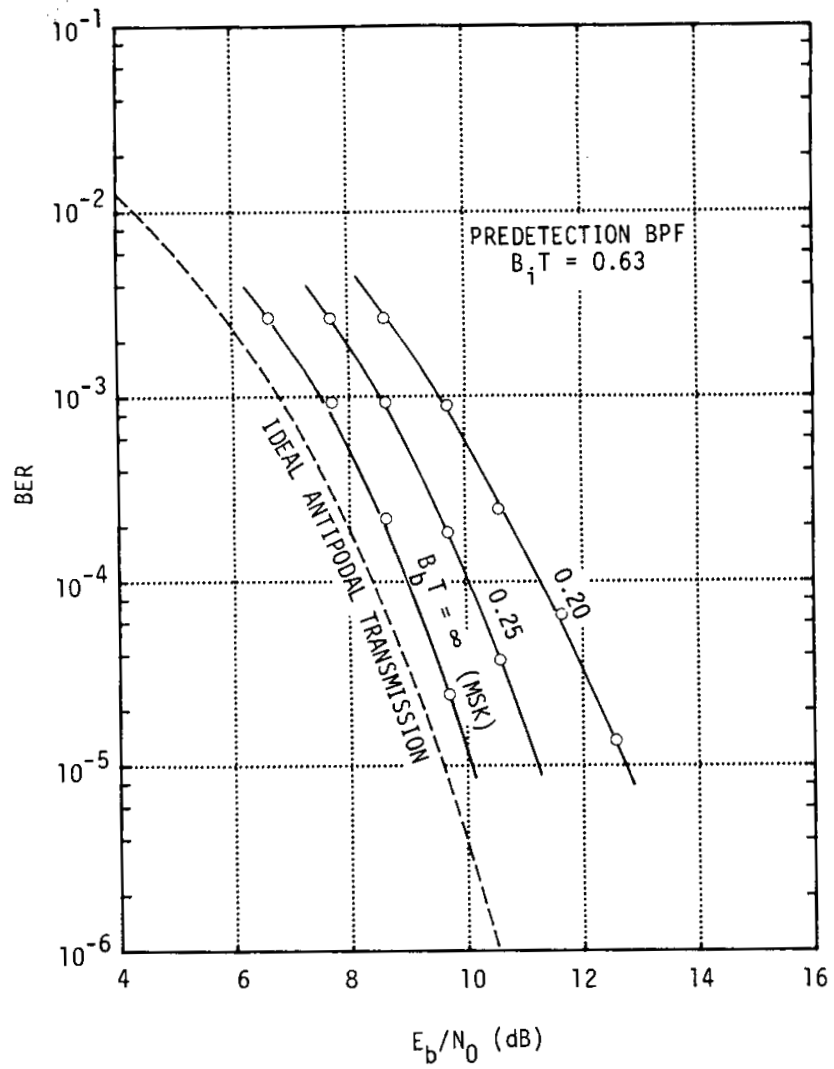

Fig. 13. Static BER performance.

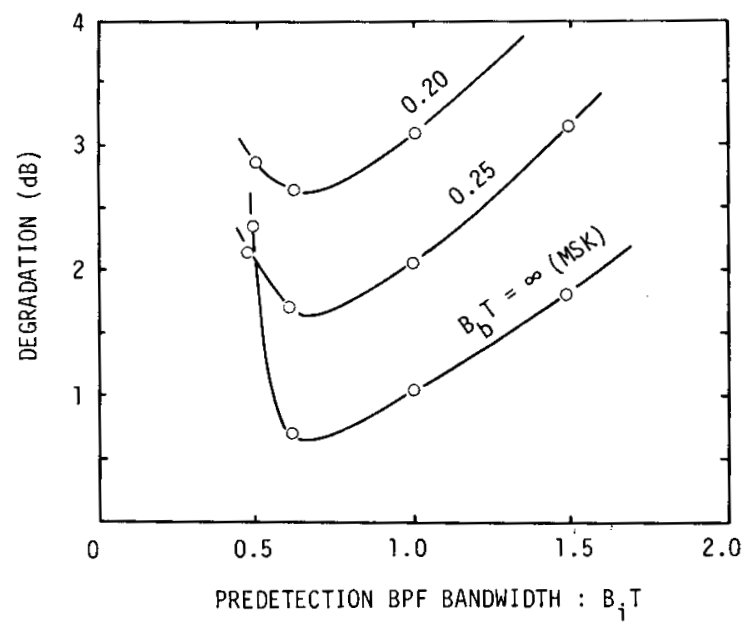

Fig. 14. Degradation of required $E_{b} / N_{0}$ for obtaining BER of $10^{-3}$.

The above-obtained results can be estimated by the degradation of the minimum signal distance shown in Figs. 5 and 6.

\section{Dynamic BER Performance}

In the practical $\mathrm{V} / \mathrm{UHF}$ land mobile radio environment, signal transmission between a fixed base station and a moving vehicle is usually performed via random multiple propagation routes. Consequently, fast and deep multipath fading, which can generally be treated by the well-known Rayleigh fading model, appears on the received signals of both stations and degrades the signal transmission performance severely.

In particular, when a quasi-stationary slow Rayleigh fading 


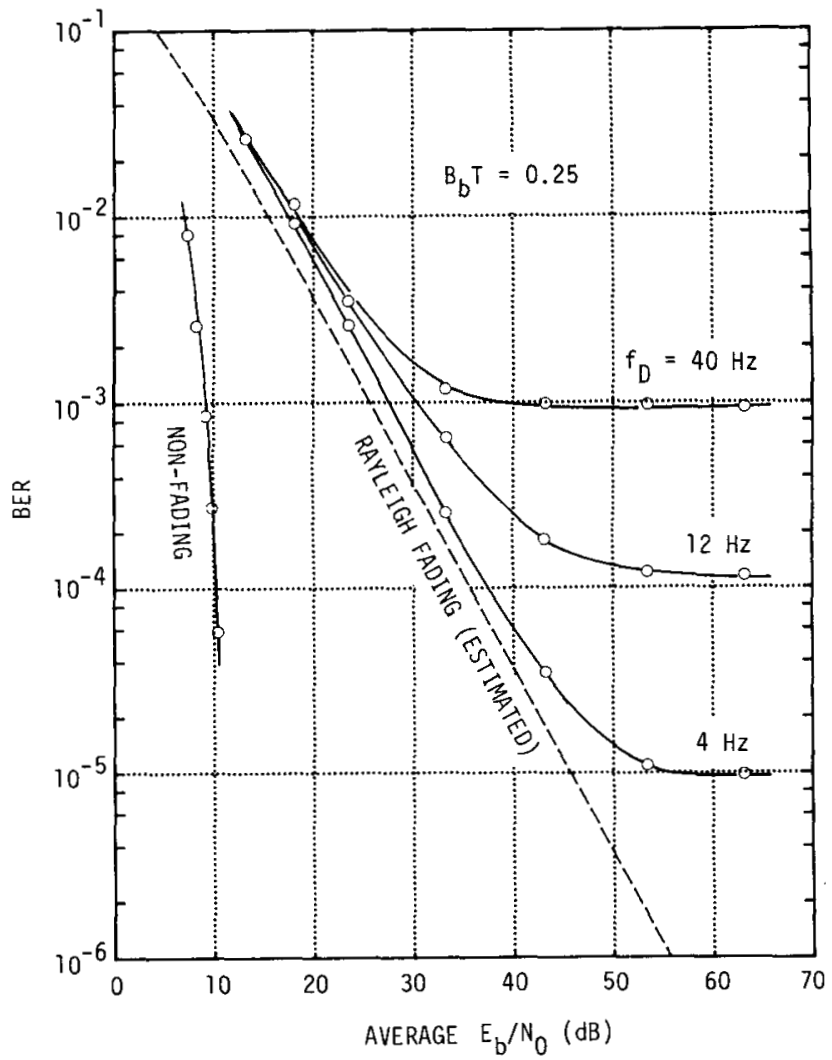

Fig. 15. Dynamic BER performance.

model is assumed, dynamic BER performance is given by

$$
P_{e}(\Gamma)=\int_{0}^{\infty} P_{e}(\gamma) p(\gamma) d \gamma
$$

where $\Gamma$ is the average $E_{b} / N_{0}$ and $p(\gamma)$ is the probability density function (pdf) of $\gamma$ given by

$$
p(\gamma)=\frac{1}{\Gamma} \exp \left(-\frac{\gamma}{\Gamma}\right)
$$

Substituting (5) and (8) into (7) yields

$$
P_{e}(\Gamma) \cong \frac{1}{2}\left(1-\sqrt{\frac{\alpha \bar{\Gamma}}{\alpha \Gamma+1}}\right) \cong \frac{1}{4 \alpha \Gamma}
$$

where $\alpha$ is the constant parameter given by (6).

However, the dynamic BER performance in the fast Rayleigh fading environment, where the temporal variation effect of the fading cannot be neglected, has not yet been theoretically estimated because the tracking performance of the carrier recovery circuit in such environment cannot be analyzed. Fig. 15 shows the experimental test results of dynamic BER performance of the GMSK with $B_{b} T=0.25$ in the simulated fast Rayleigh fading environment where the maximum Doppler frequency, i.e., the fading rate $f_{D}$, is a variable parameter. For comparison, theoretically estimated dynamic BER performance in the quasi-stationary slow Rayleigh fading environ- ment, i.e., $f_{D} T \rightarrow 0$, is also shown by the dashed line in the same figure.

\section{CONCLUSION}

As an effective digital modulation for mobile radio use, premodulation Gaussian-filtered minimum shift keying (GMSK) modulation with coherent detection has been proposed. The fundamental properties have been analyzed with the aid of machine computation. The constitution of modulator and demodulator has also been discussed. The superiority of this modulation has been supported by experimental results.

\section{ACKNOWLEDGMENT}

The authors wish to thank Dr. K. Miyauchi, S. Ito, K. Izumi, and Dr. S. Seki for their helpful guidance. They also are grateful to Dr. M. Ishizuka and H. Suzuki for their fruitful discussions.

\section{REFERENCES}

[1] O. Bettinger, "Digital speech transmission for mobile radio service," Elec. Commun., vol. 47, pp. 224-230, 1972.

[2] J. S. Bitler and C. O. Stevens, "A UHF mobile telephone system using digital modulation: Preliminary study," IEEE Trans. Vehic. Technol., vol.VT-22, pp. 78-81, Aug. 1973.

[3] N. S. Jayant, R. W. Schafer, and M. R. Karim, "Step-sizetransmitting differential coders for mobile telephony," in Proc. IEEE Int. Conf. Commun., June 1975, pp. 30/6-30/10.

[4] D. L. Duttweiler and D. G. Messerschmitt, "Nearly instantaneous companding and time diversity as applied to mobile radio transmission," in Proc. IEEE Int. Conf. Commun., June 1975, pp. $40 / 12-40 / 15$.

[5] J. C. Feggeler, "A study of digitized speech in mobile telephony." presented at the Symp. on Microwave Mobile Commun., session V-3, Boulder, CO, Sept.-Oct. 1976.

[6] H. M. Sachs, "Digital voice considerations for the land mobile radio services," in Proc. IEEE 27th Vehic. Technol. Conf., Mar. 1977. pp. 207-219.

[7] K. Hirade and M. Ishizuka, "Feasibility of digital voice transmission in mobile radio communications." Paper Tech. Group. IECE Japan, vol. CS78-2, Apr. 1978

[8] F. G. Jenks, P. D. Morgan, and C. S. Warren, "Use of four-level phase modulation for digital mobile radio." IEEE Trans. Electromagn. Compat., vol. EMC-14. pp. 113-128, Nov. 1972.

[9] P. K. Kwan, "The effects of filtering and limiting a double-binary PSK signal." IEEE Trans. Aerosp. Electron. Syst., vol. AES-5, pp. 589-594, July 1969.

[10] S. A. Rhodes, "Effects of hardlimiting on bandlimited transmission with conventional and offset QPSK modulation. ' in Proc. IEEE Nat. Telecommun. Conf., 1972, pp. 20F/1-20F/7.

[11] H. C. van den Elzen and P. van der Wurf, "A simple method of calculating the characteristics of FSK signals with modulation index 0.5," IEEE Trans. Commun., vol. COM-20, pp. 139-147, Apr. 1972.

[12] R. de Buda. "Coherent demodulation of frequency shift keying with low deviation ratio," IEEE Trans. Commun., vol. COM-20, pp. 466-470, June 1972 .

[13] H. Miyakawa et al., "Digital phase modulation scheme using continuous-phase waveform," Trans. IECE Japan, vol. 58-A, pp. 767-774, Dec. 1975.

[14] F. de Jager and C. B. Dekker. "Tamed frequency modulation, a novel method to achieve spectrum economy in digital transmission." IEEE Trans. Commun., vol. COM-20, pp. 534-542, May 1978.

[15] R. L. Didday and W. C. Lindsey, "Subcarrier tracking methods and communication system design," IEEE Trans. Commun. Technol., vol. COM-16, pp. 541-550, Aug. 1968.

[16] K. Hirade et al. "Fading simulator for land mobile radio communications," Trans. IECE Japan, vol. 58-B, pp. 449-459, Sept. 1975. 